\title{
Role of Lipid and Oxidative Stress in Psoriatic Patients - A Case Control Study
}

\author{
Roshan Kumar Jha ${ }^{1}$, Akansha Singh², Priya Koundal ${ }^{3}$, Ranjit Sidram Ambad ${ }^{4}$
}

${ }^{1}$ Department of Biochemistry, Jawaharlal Nehru Medical College, Sawangi, Meghe, Wardha, Maharashtra, India. ${ }^{2,3}$ Department of Biochemistry, Ulhas Patil Medical College and Hospital, Jalgaon, Maharashtra, India.

${ }^{4}$ Department of Biochemistry, Datta Meghe Medical College, Nagpur, Maharashtra, India.

\section{ABSTRACT}

\section{BACKGROUND}

Psoriasis is a chronic inflammatory disease. Its prevalence is around two three percent of the world populations. Categorisation of psoriasis is done on the basis of sharply demarcated dull red plaques with scales, generally on the extensor prominences and in scalp. The present study was aimed to assess the level of serum total cholesterol; triglyceride, high-density lipoprotein (HDL), low-density lipoprotein (LDL), very low-density lipoprotein (VLDL) and oxidative stress with the level of antioxidants in subjects with active psoriasis and the results were compared with healthy controls. The present study focused on evaluating oxidative stress and dyslipidaemia in psoriasis patients.

\section{METHODS}

This case and control study was conducted in a tertiary care hospital and the study period was one year. 50 cases and 50 controls were included in study followed by exclusion and inclusion criteria. Different methods were employed for evaluation of lipids and oxidative stress.

\section{RESULTS}

Parameters of lipoprotein profile were deranged and the differences were significant in psoriatic patients as compared to control group. Malondialdehyde (MDA) level was increased with significant $(\mathrm{p}<0.0001)$ difference, on the other hand, levels of glutathione peroxidase (GPx) and superoxidase dismutase (SOD) was significantly (p $<0.0001$ ) decreased, in the study group as compared to the control group.

\section{CONCLUSIONS}

Present study showed altered lipoproteins as well as oxidative stress in psoriasis patients as compared to healthy subjects. Psoriasis can cause derangement of lipid parameters and oxidative stress, and this may vary with severity of disease. Thus, hyperlipidaemia with increased peroxidation and decreased antioxidants levels are characteristic features of psoriasis.

\section{KEY WORDS}

Psoriasis, Lipoproteins, Oxidative Stress, SOD, MDA and GPx
Corresponding Author: Dr. Ranjit Sidram Ambad, Department of Biochemistry, Datta Meghe Medical College, Nagpur, Maharashtra, India. E-mail:ambad.sawan@gmail.com

DOI: $10.14260 /$ jemds $/ 2021 / 28$

How to Cite This Article: Jha RK, Singh A, Koundal P, et al. Role of lipid and oxidative stress in psoriatic patients - a case control study. J Evolution Med Dent Sci 2021;10(03):132-136, DOI: 10.14260/jemds/2021/28

Submission 09-05-2020,

Peer Review 17-11-2020,

Acceptance 24-11-2020,

Published 18-01-2021.

Copyright (C) 2021 JEMDS. This is an open access article distributed under Creative Commons Attribution License [Attribution 4.0 International (CC BY 4.0)] 


\section{BACKGROUND}

Psoriasis is a chronic inflammatory disease; its prevalence in context of world population is around two-three percentage. ${ }^{1}$ Categorisation of psoriasis is done on the basis of sharply demarcated dull red plaques with scales, generally on the extensor prominences and in scalp. Pathogenesis is not understood so far but the result from the current research shows genetic predisposition and various environmental risk factors such as stress, alcohol abuse and smoking of tobacco. These factors lead to expression of pro inflammatory cytokines and as a result of this, there is elevation in production of reactive oxygen species, which causes immune mediated skin lesions which is collectively called psoriasis. ${ }^{2}$ The metabolisms of lipids and carbohydrates are seen to be altered, perhaps, it is due to insulin resistance, its secretions and sensitivity. High incidence of cardiovascular disease (CVD) and increase in levels of oxidative stress comes together in psoriasis. It has also been seen that high prevalence of psoriasis and CVD is interconnected to each other. ${ }^{3}$ Genetic determination of psoriasis at the initial stage may be done by assessment of disorder related to lipid metabolism. ${ }^{4}$ Some of the studies done previously on psoriasis have demonstrated that psoriasis patients are more prone to non-cutaneous disease, which includes arterial and venous occlusive disease. Risk of atherosclerosis is generally increased in those people suffering from psoriasis and the cause behind this behaviour is alteration of plasma lipid and lipoprotein levels in those individuals.5,6 Numerous disorders and metabolic diseases such as hypertension, cardiovascular disease, cerebral stroke, diabetes mellitus type 2, atherosclerosis, dyslipidaemia, chronic obstructive pulmonary disease (COPD), obesity, osteoporosis, depression and cancer are sequalae to the patients with psoriasis. Most importantly, previous studies on psoriasis have concluded that there is strong correlation between lipid metabolism and pathogenesis of psoriasis. ${ }^{4,7} \mathrm{On}$ the lime light of previous study conducted on psoriasis, it has been necessary to study lipoproteins disorder and psoriasis. The outcome of study was to evaluate the level of various parameters as well as its effects on development of atherosclerosis, vascular diseases and associated mortality and morbidity.8,9

Malondialdehyde (MDA) can be employed in assessment of oxidative stress. Glutathione peroxidase (GPX) is a specific enzyme related in limiting formation of free radicals, another enzyme superoxidase dismutase (SOD) is involved in protection of cell against oxidative damage; this is a vital role of SOD, beside this, it is also useful as indicator of antioxidative status of the cell. These studies on antioxidant as well as oxidant status on the psoriasis has been done by several researchers from various geographical areas although the result was contradictory. ${ }^{10,11}$

\section{Objectives}

1. To evaluate lipoprotein and oxidative stress in psoriatic patients.

2. To estimate total cholesterol (TCH), triglycerides (TG), high density lipoprotein (HDL), low density lipoprotein (LDL), very low-density lipoprotein (VLDL), MDA, GPx, and SOD in patients with psoriasis.
3. To estimate lipid profile, MDA, GPx, and SOD in psoriatic patients and compare with Healthy Individuals.

\section{METHODS}

The case-control study was conducted in a tertiary care hospital and the study period was one year. The study was approved by institutional ethical committee (IEC) and informed consent was obtained.

\section{Inclusion Criteria}

Inclusion Criteria: Patients with psoriasis older than 18 years of age and those with psoriasis for at least 6 months from patients visiting the OPD of Dermatology, who suffer from skin disorder (psoriasis).

\section{Exclusion Criteria}

Diabetes mellitus, lactating women, pregnancy, kidney disease, patients per long term alcohol abuse history, tobacco use, hypertension, diabetes mellitus, CVD, COPD, body mass index more than $30 \mathrm{~kg} / \mathrm{m}^{2}$ or with history of metabolic disease, patients on medication affecting lipid or carbohydrate metabolism, beta blocker drugs, thiazides cyclosporins, retinoid and drugs with lipid lowering effects were excluded from the study.

\section{Study Population}

Total 100 individuals were taken for the study and divided into two groups. Group I contain control group that is healthy individuals from normal population and group II includes 50 psoriatic patients. Fifty psoriatic patients were selected from OPD of Dermatology. Sample size was calculated using Krejcie and Morgan formula.

Group I (Controls) - 50 Healthy Individuals Group II (Cases) - 50 psoriatic Patients.

\section{Biochemical Investigations}

After overnight fasting, $5 \mathrm{ml}$ of venous blood samples were collected from antecubital vein of the subjects, after written and informed consent, in dry disposable syringe under aseptic conditions and transferred to a sterile, dry and acid washed vial for biochemical investigations. Blood was allowed to clot and after formation of clot and the centrifuged supernatant was used to perform the following biochemical investigations. Haemolysed samples were discarded, and un-haemolysed sample was analysed for serum TCH, triglycerides, and HDL using enzymatic and kit methods, while VLDL and LDL were calculated using Friedwald formula. ${ }^{12,13}$

Thiobarbituric acid reactive substances (TBARs) are well established assay for screening and monitoring lipid peroxidation. The rapid and easy protocol has been modified by researchers in the evolution of drugs, food as well as human and animal tissue samples. MDA forms as 1:2 adduct with TBA. The MDA-TBA adduct formed from the reaction of MDA samples with TBA which can be measured calorimetrically. 
TBARs levels are determined from an MDA estimation equivalence standard 1, 1, 3, 3-tetraethoxy propane. ${ }^{14}$ GPx catalyses the reaction of glutathione by cumene hydroperoxide to reduced form which is measured at $340 \mathrm{~nm} .{ }^{3}$

SOD principle was based on reaction of xanthine oxidase (XOD) with superoxide radicles that reacts with $2-4$ iodophenyl 3 - 4 nitrophenyl-5 phenyltetrazolium chlorides to form a red formazan dye. ${ }^{15}$

\section{Statistical Analysis}

All estimated results were expressed as mean \pm SD. Mean values will be assessed for significance by unpaired student- $t$ test. A statistical analysis was performed using the Statistical Package for the Social Science program (SPSS, 24.0). Frequencies and percentages will be used for the categorical measures. Probability values $\mathrm{p}<0.05$ was considered statistically significant.

\section{RESULTS}

\begin{tabular}{|cccc|}
\hline Parameters & Group I (Controls) & $\begin{array}{c}\text { Group II } \\
\text { (Cases) }\end{array}$ & P-Value \\
\hline Total cholesterol (mg \%) & $145.89 \pm 20.5$ & $221.8 \pm 26.6$ & $\mathrm{P}<0.0001$ \\
Triglyceride (mg \%) & $129.4 \pm 16.10$ & $220.12 \pm 16.0$ & $\mathrm{P}<0.0001$ \\
\hline VLDL (mg \%) & $25.88 \pm 4.23$ & $44.02 \pm 4.51$ & $\mathrm{P}<0.0001$ \\
\hline LDL (mg \%) & $65.89 \pm 12.98$ & $140.27 \pm 10.81$ & $\mathrm{P}<0.0001$ \\
\hline HDL (mg \%) & $54.12 \pm 4.32$ & $37.51 \pm 4.68$ & $\mathrm{P}<0.0001$ \\
\hline Table 1. Lipid Profile in Patients with Psoriasis \\
\hline \multicolumn{4}{|r}{} \\
\hline \multicolumn{4}{|l}{} \\
\hline
\end{tabular}

Table 1 depicts there is significant differences in the various parameters of lipids in patients of psoriasis as compared to that with normal subjects.

\begin{tabular}{|cccc|}
\hline Parameters & $\begin{array}{c}\text { Group I } \\
\text { (Controls) }\end{array}$ & Group II (Cases) & P-Value \\
\hline $\mathrm{MDA}(\mathrm{nmol} / \mathrm{mL})$ & $0.93 \pm 0.21$ & $2.65 \pm 0.30$ & $\mathrm{P}<0.0001$ \\
$\mathrm{SOD}(\mathrm{U} / \mathrm{mgHb})$ & $8.83 \pm 0.8$ & $7.19 \pm 0.41$ & $\mathrm{P}<0.0001$ \\
$\mathrm{GPx}(\mathrm{U} / \mathrm{mgHb})$ & $15.64 \pm 1.43$ & $14.27 \pm 0.33$ & $\mathrm{P}<0.0001$ \\
\hline \multicolumn{4}{|c|}{ Table 2. Levels of s-Oxidants and Antioxidants Status } \\
& in Patients with Psoriasis & \\
\hline
\end{tabular}

Table 2 depicts the level of MDA was statistically significant $(\mathrm{p}<0.0001)$ in cases as compared to controls, on the other side the levels of GPx and SOD antioxidants were significantly decreased $(\mathrm{p}<0.0001)$ in cases as compared to controls. (Table 2).

\section{DISCUSSION}

Psoriasis is not a new disease; its history is thousand years old. Study of lipids, its metabolic disorder and its interrelation with chronic disease psoriasis has been started since beginning of $20^{\text {th }}$ century. ${ }^{7}$ Numerous studies have been concentrated on skin surface lipids, lipids from stratum corneum, phospholipids present on epidermis, dermal LDL in psoriatic skin, lipid metabolism as well as oxidative stress and its correlation with clinical features, symptoms of psoriasis, parameters to assess inflammation and parameters of lipoprotein metabolism. One of the previous studies suggested describing the psoriasis as an immuno-metabolic syndrome because of the association of lipid and immunologic abnormalities observed in psoriasis. ${ }^{16}$

Dyslipidaemia and psoriasis are controversial to some extent, because of contradictory findings. Serum lipoproteins have been assessed by many study groups and were compared to relevant healthy controls. 4,5,9,17 Some of the studies suggest that blood lipids are significantly dependent on group matching which includes age, sex, ethnic and cultural factors. Several studies show statistically significant increased level of total cholesterol, LDL and triglycerides in the patients suffering with psoriasis when the parameters were compared with healthy patients. ${ }^{4,5,8,9,17-20}$ In the context of present study results were similar to above findings where the levels of total cholesterol, triglycerides and LDL were significantly increased $(\mathrm{P}<0.0001)$. On the other side, there was significant decrease in the levels of high-density lipoprotein (HDL) among cases as compared to control group of healthy people.20,21,22 Few studies conducted on psoriasis differ from present study findings where serum lipid levels between psoriatic patients and control made no differences. ${ }^{23,24}$ Present study shows significant differences in the levels of lipoproteins.

There are several mechanisms supporting relationship between elevated levels of lipoproteins and psoriasis. Psoriasis has been now considered as a systemic inflammatory disease with type 17 helper T cells (TH-1), type 17 helper T cells (TH-17) and inflammatory cytokines contributing to its pathogenesis. ${ }^{9}$ In contrast to this finding, psoriasis is interrelated with lipids and immunological abnormalities that is why the disease is described as an immuno metabolic syndrome. ${ }^{25}$

Biomolecules, cell membrane and tissues may get damaged due to over production or increased production of free radicals. Oxidation of polyunsaturated fatty acid results in the formation of products yielding from lipid peroxidation such as MDA.

Present Study shows levels of MDA in psoriasis are increased significantly as compared to controls. The finding of present study is somehow comparable to study conducted by Pereira et al. as well as Relhan V et al. ${ }^{26,27}$ While, a few studies did not observe similar findings, Yildrium et al., study did not show any correlation with level of MDA in psoriatic cases as compared to controls. ${ }^{28}$

Lipid peroxidation has an interesting mechanism in psoriatic patients; due to increase in reactive oxygen species (ROS) production which diminishes antioxidant level, increases oxidative stress and at last causes lipid peroxidation. It possibly will be causing cell damage through continuous chain reactions. It also aids phospholipase A2 activation, initiate secretion of many mediators by arachidonate, in deactivation of adenylate cyclase and activation of guanyl cyclase which decreases cyclic adenosine monophosphate /cyclic guanosine monophosphate (cAMP / cGMP) ratio and that is associated to epidermal proliferation in psoriasis patients. 3,9

Present study observed significant $(\mathrm{p}<0.0001)$ decrease in levels of SOD [Table 2]. Literatures have observed in patients with psoriasis there is increased capability of activating polymorphonuclear neutrophils (PMNs) which encourage elevation of superoxide anion $\left(\mathrm{O}_{2+}\right)$. Increased generation of super oxide anions from neutrophils and neutrophil accumulation in lesions caused due to psoriasis, and thus the phagocytic reactions results in production of SOD. 
Early and active psoriatic lesion is characterised by means of the intra-epidermal polymorphonuclear leukocytes (PMNL) penetration. Thus, superoxide plays a significant role. ${ }^{3}$ Superoxide dismutase is an antioxidant enzyme which catalyses dismutation of superoxide to lesser harmful molecules, oxygen and hydrogen peroxide.

Beside the above literatures, present findings are similar to Yildrium et al., Pujari et al. and kobayashi et al. study.28,29,30 While results observed by some studies were not similar to the present study findings. ${ }^{11,31}$ Although, present findings observed significant decrease in levels of SOD in psoriatic patients, which may be used to counter act the stress caused by oxidation.

Glutathione peroxidase (GPx) is present in cells; it is involved in alteration of hydroperoxide group to much less toxic hydroxyl product. Present study observed significant (p $<0.0001$ ) decrease in levels of GPx in patients with psoriasis as compared to normal healthy subjects. (Table 2). Study conducted by Kokcam et al. observed similar finding to present study where they observed GPx levels were significantly decreased in patients with psoriasis as compared to normal healthy subjects. ${ }^{32}$ Selenium is believed as an essential part of enzyme, decrease in GPx activity indicates that the hydroxyl radicles are gathering in exacerbated tissue. A study conducted by Michaelson et al. and Farris et al. observed selenium (Se) supplementation have positive effect on acne, ultraviolet (UV) induced inflammation in bald mice, also reported decrease in level of Se in psoriatic patients. ${ }^{32,33,34,35}$

\section{CONCLUSIONS}

Present study showed altered lipoproteins as well as oxidative stress in psoriatic patients as compared to healthy subjects. The present study concludes that the hyperlipidaemia with increased peroxidation and decreased antioxidant levels are characteristic features of psoriasis. Inactivation of free radicles and stabilization to damage of cell membranes can be achieved by supplementation, which may be used for established therapeutic approach in future.

The present study had a smaller number of cases enrolled and no proper follow up was taken. There is a need for intervention with supplementation to evaluate the effect of supplementation on the management of psoriasis.

Data sharing statement provided by the authors is available with the full text of this article at jemds.com.

Financial or other competing interests: None.

Disclosure forms provided by the authors are available with the full text of this article at jemds.com.

\section{REFERENCES}

[1] Aggarwal J, Singh A, Ahmed TI, et al. Altered levels of serum $\mathrm{Cu}$ and $\mathrm{Cu} / \mathrm{Zn}$ ratio in psoriasis. International Journal of Current Medical Science and Practice 2019;24(1-2):25-8.

[2] Aggarwal J, Singh A, Gupta S, et al. Copper and zinc status in psoriasis: correlation with severity. Indian Journal of Clinical Biochemistry 2020:1-4.
[3] Gupta M, Chari S, Borkar M, et al. Dyslipidemia and oxidative stress in patients of psoriasis. Biomedical Research 2011;22(2):222-5.

[4] Mallbris L, Granath F, Hamsten A, et al. Psoriasis is associated with lipid abnormalities at the onset of skin disease. J Am Acad Dermatol 2006;54(4):614-21.

[5] Akhyani M, Ehsani AH, Robati RM, et al. The lipid profile in psoriasis: a controlled study. J Eur Acad Dermatol Venereol 2007;21(10):1330-2.

[6] Pietrzak A, Lecewicz-Toruń B. Activity of serum lipase (EC 3.1.1.3) and the diversity of serum lipid profile in psoriasis. Med Sci Monit 2002;8(1):CR9-13.

[7] Pietrzak A, Michalak-Stoma A, Chodorowska G, et al. Lipid disturbances in psoriasis: an update. Mediators Inflamm 2010;2010:535612.

[8] Piskin S, Gurkok F, Ekuklu G, et al. Serum lipid levels in Psoriasis. Yonsei Med J 2003;44(1):24-6.

[9] Javidi Z, Meibodi NT, Nahidi Y. Serum lipids abnormalities and psoriasis. Indian J Dermatol 2007;52(2):89-92.

[10] Ghosh A, Mukhopadhyay S, Kar M. Role of free reactive iron in Psoriasis. Indian J Dermatol Venereol Leprol 2008;74(3):277-8.

[11] Baz K, Cimen MYB, Kokturk A. Oxidant/antioxidant status in patients with psoriasis. Yonsei Med J 2003;44(6):98790.

[12] Nakhwa YC, Rashmi R, Basavaraj KH. Dyslipidemia in psoriasis: a case controlled study. Int Sch Res Notices 2014;2014:729157.

[13] Mahajan S, Ambad RS, Jha RK, et al. To estimate lipid profile in patients with COPD. International Journal of Medical and Biomedical Studies 2020;4(1):879.

[14] Satoh K. Serum lipid peroxide in cerebrovascular disorders determined by a new colorimetric method. Clin Chem Acta 1978;90(1);37-43.

[15] Das K, Samanta L, Chainy GBN. A modified spectrophotometeric assay of superoxide dismutase using nitrite formation by superoxide radical. Inidan Journal of Biochemistry and Biophysics 2000;37(3):2014.

[16] Bhat RM, Hyacinth P. Lipid profile in psoriasis patients. Psoriasis: Targets and Therapy 2012;2:77-80.

[17] Tekin NS, Tekin IO, Barut F, et al. Accumulation of oxidized low-density lipoprotein in psoriatic skin and changes of plasma lipid levels in psoriatic patients. Mediators Inflamm 2007;2007:78454.

[18] Gisondi P, Tessari G, Conti A, et al. Prevalence of metabolic syndrome in patients with psoriasis: a hospital-based case-control study. Br J Dermatol 2007;157(1):68-73.

[19] Amin T, Saied E, Abdou SH. Atherosclerotic risk in psoriasis. Journal of Pan-Arab League of Dermatologists 2005;16(2):39-45.

[20] Bajaj DR, Mahesar SM, Devrajani BR, et al. Lipid profile in patients with psoriasis presenting at Liaquat University Hospital Hyderabad. J Pak Med Assoc 2009;59(8):512-5.

[21] Örem A, Çimşit G, Değer 0, et al. The significance of autoantibodies against oxidatively modified low-density lipoprotein (LDL) in patients with psoriasis. Clin Chim Acta 1999;284(1):81-8. 
[22] Kural BV, Örem A, Çimşit G, et al. Evaluation of the atherogenic tendency of lipids and lipoprotein content and their relationships with oxidant-antioxidant system in patients with psoriasis. Clin Chim Acta 2003;328(12):71-82.

[23] Farshchian M, Zamanian A, Farshchian M, et al. Serum lipid level in Iranian patients with psoriasis. J Eur Acad Dermatol Venereol 2007;21(6):802-5.

[24] Toker A, Kadı M, Yıldırım AK, et al. Serum lipid profile paraoxonase and arylesterase activities in psoriasis. Cell Biochem Funct 2009;27(3):176-80.

[25] Ahmed AA. Serum lipid profile in psoriasis: a controlled study. Tikrit Medical Journal 2011;17(1):38-42.

[26] Rocha-Pereira P, Santos-Silva A, Rebelo I, et al. Erythrocyte damage in mild and severe psoriasis. Br J Dermatol 2004;150(2):232-44.

[27] Relhan V, Gupta SK, Dayal S, et al. Blood thiols and malondialdehyde levels in psoriasis. J Dermatol 2002;29(7):399-403.

[28] Yildirim M, Inaloz HS, Baysal V, et al. The role of oxidants and antioxidants in psoriasis. J Euro Acad Dermatol Venereol 2003;17(1):34-6.
[29] Pujari VM, Suryakar AN, Ireddy S. Oxidants and antioxidant status in psoriasis patients. Biomed Res 2010;21(2):221-4.

[30] Kobayashi T, Matsumoto M, Iizuka H, et al. Superoxide dismutase in psoriasis, squamous cell carcinoma and basal cell epithelioma: an immunohistochemical study. $\mathrm{Br}$ J Dermatol 1991;124(6):555-9.

[31] Utaş S, Köse K, Yazici C, et al. Antioxidant potential of propylthiouracil in patients with psoriasis. Clin Biochem 2002;35(3):241-6.

[32] Kökçam I, Nazıroğlu M. Antioxidants and lipid peroxidation status in the blood of patients with psoriasis. Clin Chim Acta 1999;289(1-2):23-31.

[33] Fuchs J, Huflejt ME, Rothfuss LM, et al. Impairment of enzymic and nonenzymic antioxidants in skin by UVB irradiation. J Invest Dermatol 1989;93(6):769-73.

[34] Michaelsson G, Berne B, Carlmark B, et al. Selenium in whole blood and plasma is decreased in patients with moderate and severe psoriasis. Acta Derm Venereol 1989;69(1):29-34.

[35] Fairris GM, Perkins PJ, Lloyd B, et al. The effect on atopic dermatitis of supplementation with selenium and vitamin E. Acta Derm Venereol 1989;69(4):359-62. 